\title{
Locoregional tumor failure after definitive radiation for patients with stage III non-small cell lung cancer
}

\author{
Raj S Rajpara, Eduard Schreibmann, Tim Fox, Liza J Stapleford, Jonathan J Beitler, Walter J Curran \\ and Kristin A Higgins ${ }^{*}$
}

\begin{abstract}
Background: Locoregional tumor failure (LRF) after definitive chemoradiation for patients with stage III NSCLC remains unacceptably high. This analysis sought to further define where LRF occurs relative to radiation dose received and pre-treatment PET scan-defined maximum standard uptake value (SUVmax).

Methods: This was a retrospective study analyzing patients with stage III NSCLC treated with definitive radiation between 2006 and 2011. LRF was defined as failure within the ipsilateral lung, hilum or mediastinum. The CT simulation scan with the radiation dose distribution was registered to the CT or PET/CT documenting LRF. The region of LRF was contoured, and the dose to $95 \%$ of the volume (D95) of LRF was extracted. The pre-treatment SUVmax was also extracted for the anatomic region of LRF.

Results: Sixty-one patients were identified. Median follow-up time was 19.1 months (range 2.37-76.33). Seventy four percent of patients were treated with 3-D conformal technique (3DCRT), 15\% were treated with Intensity Modulated Radiotherapy (IMRT), and 11\% were treated with a combination of 3DCRT and IMRT. Median prescribed radiation dose for all patients was 66 Gy (39.6-74). Concurrent chemotherapy was delivered in $90 \%$ of patients. Twenty-two patients (36\%) developed a LRF, with a total of 39 anatomic regions of LRF identified. Median time to LRF was 11.4 months (3.5-44.6). Failures were distributed as follows: $36 \%$ were in-field failures, $27 \%$ were out-of-field failures, $18 \%$ were in-field and out-of-field failures, and $18 \%$ were in-field and marginal (recurrences within the field edge) failures. There were no isolated marginal failures. Of the patients that developed a LRF, 73\% developed a LRF with an in-field component. Sixty-two percent of LRFs were nodal. The median pre-treatment SUVmax for the anatomic region of LRF for patients with an in-field failure was 13. The median D95 of in-field LRF was 63 Gy.

Conclusions: LRF after definitive chemoradiation are comprised primarily of in-field failures, though out-of field failures are not insignificant. Marginal failures are rare, indicating field margins are appropriate. Although radiation dose escalation to standard radiation fields has not yielded success, using PET parameters to define high-risk regions remains worthy of further investigation.
\end{abstract}

Keywords: Lung cancer, Locally advanced, Locoregional failure, PET/CT, Radiation, Chemotherapy

\footnotetext{
*Correspondence: kristin.higgins@emory.edu

Department of Radiation Oncology, Emory University School of Medicine and Winship Cancer Institute of Emory University, Atlanta, GA 30322, USA
} 


\section{Background}

Lung cancer continues to be the leading cause of cancer death in the United States [1]. In 2013, an estimated 228,000 new cases of lung cancer were diagnosed and 160,000 lung cancer-related deaths were estimated to have occurred [1]. Non-small cell lung cancer (NSCLC) accounts for $80 \%$ of all lung cancers, and approximately $30-40 \%$ of patients with NSCLC present with unresectable, locally advanced disease. Concurrent chemoradiation was established as the standard of care for patients with inoperable non-small cell lung cancer (NSCLC) by several randomized trials performed in the 1990s that demonstrated an overall survival benefit with the delivery of concurrent as compared to sequential chemoradiation [2-4]. Despite the survival benefit gained from concurrent chemoradiation therapy, clinical outcomes, particularly locoregional control, remain suboptimal. Previous studies have demonstrated local control rates of $50-70 \%$, but some have reported locoregional control rates as low as $15 \%[2,3,5,6]$. Achieving local control of disease in NSCLC is important, as a recent meta-analysis demonstrated local control significantly improves overall survival results [5].

The use of positron emission tomography/computed tomography (PET/CT) with 2-[ $\left.{ }^{18} \mathrm{~F}\right]$ fluoro-2-deoxy-Dglucose (FDG) for staging patients with NSCLC has increased over the past two decades and is now considered standard of care in the diagnostic workup in patients with newly diagnosed NSCLC. PET/CT has been shown to have better sensitivity in detecting metastatic disease and to significantly reduce the size of radiation treatment volumes [7-9]. Another potential advantage of PET/CT with FDG is the ability to quantitatively analyze results by using the standardized uptake value (SUV). The SUV of the primary tumor has been shown to correlate with prognosis in NSCLC and may be a marker of disease activity $[10,11]$. The predictive value of PET/CT is still undefined, however PET/CT presents an opportunity to develop imaging biomarkers to predict locoregional failure (LRF) and thus refine delivery of radiotherapy in patients with unresectable, non-metastatic NSCLC.

The present study analyzed LRF relative to radiation treatment fields and radiation dose received, in an effort to understand the adequacy of planning treatment volumes and to define anatomic regions most at risk for LRF. Additionally, the pretreatment SUVmax of regions of LRF were analyzed to determine if these regions had a higher SUVmax relative to anatomic regions without LRF.

\section{Methods}

An Emory University Institutional Review Board approved study was performed on newly diagnosed, stage III NSCLC patients undergoing curative intent radiotherapy between 2006 and 2011. ICD-9 codes were used to identify patients with lung cancer treated with radiotherapy between 2006 and 2011. Inclusion criteria included patients with AJCC ( $7^{\text {th }}$ edition) Stage IIIA and Stage IIIB, newly diagnosed, histologically confirmed NSCLC treated with curative intent radiotherapy. Patients included in this study were treated at Emory University Hospital or Emory University Hospital Midtown. Patients with disease other than stage III were excluded, as were stage III patients treated with surgery and postoperative radiation or patients treated with preoperative chemoradiation. Patients without pre-treatment PET/CT imaging or post-treatment (PET/CT or chest CT) imaging at Emory University were excluded from this study.

Clinical characteristics of patients were extracted from the electronic medical chart, including age, sex, race, histology, $\mathrm{T}$ stage, $\mathrm{N}$ stage, and administration of chemotherapy. Details of radiation treatment, including dose, treatment technique, and use of daily on-board imaging were obtained through the radiation oncology electronic chart as well as radiation oncology treatment planning software.

\section{Defining and analyzing locoregional tumor failures}

Locoregional tumor failure was defined as recurrent or persistent disease involving the ipsilateral lung, hilum, or mediastinum. Radiology reports and radiology examinations including CT and/or PET/CT were reviewed to determine LRF. Biopsy was not required for confirmation of LRF if serial imaging confirmed persistent or recurrent disease. Supraclavicular recurrences and contralateral lung recurrences were scored as distant failures. Locoregional failures were included as either a first failure event or coincident with distant failure.

For patients who developed a LRF, the CT simulation scan with radiation dose distribution was registered to either the post-treatment $\mathrm{CT}$ or $\mathrm{PET} / \mathrm{CT}$ that documented a LRF. A commercial deformable image registration and dose mapping software (VelocityAI, Velocity Medical Solutions, Atlanta, GA), was used to register the two image studies and extract both dose and SUVmax from the region of interest. The deformable registration algorithm uses free-form deformations based on cubic spline interpolation between sparse, uniformly distributed control points as its transformation model and has been previously described in detail [12-14]. The anatomic region of LRF within the lung, mediastinum, or hilum was contoured, using characteristics including soft-tissue fullness, contrast enhancement, asymmetry and FDG avidity to distinguish tumor from normal, adjacent tissue. Recurrences involving lung parenchyma were contoured on a lung window and recurrences involving soft tissue were contoured on a soft tissue window. A dose volume histogram was created to determine the dose to 95\% (D95) of the volume of LRF. Locoregional failures were determined 
to be in-field (within the 95\% isodose line), out-of-field (greater than $2 \mathrm{~cm}$ outside the $95 \%$ isodose line), or marginal (within $2 \mathrm{~cm}$ of the $95 \%$ isodose line) based on the relation of the LRF region to the anatomic region encompassed by the radiation fields.

\section{Integrating Pre-treatment PET/CT}

The pre-treatment PET/CT was used to determine the pre-treatment SUVmax of the anatomic region of LRF. SUVmax was defined as the maximum SUV to a voxel location contained within the primary tumor and the involved lymph nodes. The region of LRF was contoured, using the methods described above, on the CT scan documenting LRF. Using the previously described deformable image registration software (VelocityAI), the posttreatment CT scan was registered to the pre-treatment $\mathrm{PET} / \mathrm{CT}$ scan, and the pre-treatment SUVmax was extracted in the region of LRF. A single user (R.R) performed all image registrations, contouring of LRF, and SUVmax determination.

The SUVmax was quantitatively used to determine FDG-PET activity. SUV was defined as normalizing the tumor uptake of FDG to injected dose per body weight of the patient. SUVmax was defined as the maximum SUV value at a pixel in the volume of interest and was calculated by the image registration software (VelocityAI) from the absolute activity $(\mathrm{Bq} / \mathrm{mL})$ in each voxel.

Descriptive statistics were used to illustrate the patient population. Student $t$ - tests were used to compare median pre-treatment SUVmax values for patients with LRF versus those patients without $L R F$, and to compare pretreatment SUVmax values in anatomic regions of LRF relative to pre-treatment SUVmax values in patients without LRF.

\section{Results}

\section{Patient characteristics}

Sixty-one patients met inclusion criteria for this study. Patient and treatment characteristics are summarized in Table 1. The median age at diagnoses was 65 (range 40-84). Median follow-up time was 19.1 months (range 2.476.3 months). The majority of patients (64\%) presented with Stage IIIB disease. The most common histology was adenocarcinoma, consisting of $49 \%$ of cases. Concurrent chemotherapy was administered in $90 \%$ of patients. A platinum-based doublet regimen was administered to all patients receiving concurrent chemotherapy. Carboplatin was given with paclitaxel (42 patients, 76\%), pemetrexed (4 patients, 7\%), and etoposide (1 patient, 2\%). Cisplatin was given with etoposide (6 patients, $11 \%$ ), pemetrexed (1 patient, 2\%), and docetaxel (1 patient, 2\%). Six patients received radiotherapy alone. The median radiation dose was 66 Gy (range 39.6-74 Gy). The median pretreatment
Table 1 Patient and treatment characteristics

\begin{tabular}{|c|c|}
\hline Characteristics & Patients, $n=61$ \\
\hline Median age (range) & $65(40-84)$ \\
\hline \multicolumn{2}{|l|}{ Sex } \\
\hline Male & $29(48 \%)$ \\
\hline Female & $32(53 \%)$ \\
\hline \multicolumn{2}{|l|}{ Race } \\
\hline White & $39(64 \%)$ \\
\hline Black & $21(34 \%)$ \\
\hline Other & $1(2 \%)$ \\
\hline \multicolumn{2}{|l|}{ Histology } \\
\hline Adenocarcinoma & $30(49 \%)$ \\
\hline Squamous & $18(30 \%)$ \\
\hline NSCLC NOS* & $13(21 \%)$ \\
\hline \multicolumn{2}{|l|}{ T Stage } \\
\hline $\mathrm{T} 1$ & $9(15 \%)$ \\
\hline $\mathrm{T} 2$ & $20(33 \%)$ \\
\hline T3 & $11(18 \%)$ \\
\hline T4 & 19 (31\%) \\
\hline Tx & $2(3 \%)$ \\
\hline \multicolumn{2}{|l|}{ N Stage } \\
\hline No & $1(2 \%)$ \\
\hline N2 & $32(52 \%)$ \\
\hline N3 & $28(46 \%)$ \\
\hline \multicolumn{2}{|l|}{ AJCC Stage (V.7) } \\
\hline$\| \mathrm{A}$ & $22(36 \%)$ \\
\hline$\| \mathrm{IIB}$ & 39 (64\%) \\
\hline \multicolumn{2}{|l|}{ Radiation Technique } \\
\hline 3-D Conformal & $45(74 \%)$ \\
\hline IMRT & $9(15 \%)$ \\
\hline 3-D Conformal \& IMRT & $7(11 \%)$ \\
\hline Radiation Dose, median (range) & 66 Gy (39.6-74 Gy) \\
\hline \multicolumn{2}{|c|}{ Treatment Planning Margins, median (range) } \\
\hline GTV to CTV expansion & $0.5 \mathrm{~cm}(0-1 \mathrm{~cm})$ \\
\hline CTV to PTV expansion & $1 \mathrm{~cm}(0.3-2.5 \mathrm{~cm})$ \\
\hline Use of daily On-board Imaging (OBI) & $24(39 \%)$ \\
\hline Concurrent Chemotherapy & $55(90 \%)$ \\
\hline
\end{tabular}

*NSCLC NOS- non-small cell carcinoma not otherwise specified.

SUVmax of the primary tumor and involved lymph nodes for the entire patient cohort was 15.2 (range 6.6-50.1).

\section{Treatment characteristics}

All patients underwent CT-based radiation planning. All patients underwent a pretreatment PET/CT that was used to define radiation treatment volumes. Seventy-four percent of patients were treated with 3-D conformal radiotherapy (3DCRT), 15\% were treated with IMRT, and 11\% were 
treated with both 3DCRT and IMRT. Of the 45 patients treated with 3DCRT, 35 (78\%) patients were treated with anterior-posterior (AP) and posterior-anterior (PA) fields followed by off-cord oblique fields. Seven, 8, or 9-field IMRT was used for the patients treated with IMRT technique. The median gross tumor volume (GTV) to clinical target volume (CTV) margin used was $0.5 \mathrm{~cm}$ (range 0-1.0 cm). The median CTV to planning target volume (PTV) margin used was $1 \mathrm{~cm}$ (range 0.3-2.5 cm). Elective nodal radiation of non-metabolically active nodal stations was generally not performed. Heterogeneity corrections for treatment planning were used in $31 \%$ of patients. Onboard imaging, consisting of KV (kilovoltage) orthogonal films, was used in $39 \%$ of patients.

\section{Locoregional tumor failure analysis}

Twenty-two patients developed a LRF. The median time to a LRF was 11.4 months (range 3.5-44.6 months). The median radiation dose for patients that developed a LRF was 66 Gy (range 45-66.6 Gy). The median pre-treatment SUVmax of the primary tumor and lymph nodes was 14.9 (range 7.9-50.1) in patients with LRF, but was not significantly different compared with the median pre-treatment SUVmax (15.8, range 6.6-38.6) of patients without LRFs. Five patients developed isolated LRF whereas 17 patients developed LRF and distant failure. Of the 22 patients who developed a LRF, 8 patients developed isolated in-field failures, 6 patients developed isolated out-of-field failures, 4 patients developed in-field and out-of-field failures, and 4 patients developed in-field and marginal failures (Table 2, Figures 1, 2 and 3). No patient developed a marginal failure alone. In total, $73 \%$ of patients with a LRF had an in-field component of recurrence. Isolated out-of-field failures occurred in $27 \%$ of patients that developed a LRF. Of the out-field recurrences, three patients developed out-of-field nodal recurrences, two patients developed outof-field lung recurrences, and one patient developed both an elective nodal failure and out of field lung recurrence. Thus, LRF in elective nodal regions occurred in $7 \%$ of the entire patient cohort.

A total of 39 anatomic regions of failure were identified in the 22 patients that developed a LRF. Fifteen (38\%) failures occurred within the parenchyma of the lung and $62 \%$ occurred in regional lymph nodes. A total

\begin{tabular}{ll}
$\begin{array}{l}\text { Table } \mathbf{2} \text { Patient specific local regional failures in relation } \\
\text { to radiation treatment fields }\end{array}$ \\
\hline Location of local regional failure & $\mathbf{n = 2 2 ~ ( 3 6 \% )}$ \\
\hline In-Field & $8(36 \%)$ \\
In-field and Marginal & $4(18 \%)$ \\
Out-of-field & $6(27 \%)$ \\
In-field and Out-of-field & $4(18 \%)$ \\
Marginal (isolated) & $0(0 \%)$ \\
\hline
\end{tabular}

of $22(56 \%)$ anatomic regions of failures occurred in field, 13 failures occurred out of field, and 4 failures occurred in the field edge (Table 3). The median pretreatment SUVmax for anatomic regions with an in-field failure was 13.4 (range 4.8-43) and was not statistically different from the median SUVmax of patients without a LRF (15.8, range 6.6-38.6). The median D95 to the anatomic region that failed in-field was 63.4 Gy (range 21.3$69.5 \mathrm{~Gy}$ ). Of the 22 anatomic regions of in-field failure, 12 failures (55\%) occurred in the treated mediastinum and 10 failures occurred within the treated lung parenchyma. In the 8 patients with isolated in-field LRFs, a total of 11 anatomic regions were identified; 6 failures occurred within the treated mediastinum and 5 within the treated lung parenchyma. In the 6 patients with isolated out-of-field LRFs, a total of 8 anatomic regions were identified; 5 within the untreated mediastinum, and 3 within the untreated lung parenchyma. Isolated out-offield LRFs accounted for $21 \%$ of all anatomic regions of failure.

\section{Discussion}

Locoregional tumor failure after definitive chemoradiation for Stage III NSCLC remains high despite contemporary radiation techniques and the addition of concurrent chemotherapy. A 36\% crude LRF rate was found in this study, which is comparable to previous reports $[2,3,5]$. Interestingly, in this study, despite the use of PET/CTbased planning and a median radiation dose of $66 \mathrm{~Gy}$, LRF after definitive radiation is primarily an in-field event (73\%). The marginal failure rate in this study is low, indicating that the GTV to CTV and CTV to PTV margin expansions used in this study are likely appropriate to account for microscopic extent of disease and treatment setup error and tumor motion, respectively.

In-field LRF was not due to inadequate dose, as the median D95 to the anatomic region of LRF was $63 \mathrm{~Gy}$, indicating the region of LRF did receive a tumoricidal dose of radiation. This median dose to regions of LRF is similar to that mandated to the PTV in clinical trials, which typically require $95 \%$ of the PTV to receive $100 \%$ of the prescription dose. Thus, these anatomic regions where in-field LRF is occurring likely represent radioresistant disease. Strategies to overcome radiation resistance continue to evolve. The Radiation Therapy Oncology Group (RTOG) is currently examining dose escalation to persistently metabolically active disease defined by in-treatment FDG PET (RTOG 11-06). Other strategies under investigation include the addition of molecular agents to standard chemoradiation that target radioresistant pathways.

Curing patients with locally advanced NSCLC is not possible without locoregional disease control. Improving local control has been shown to impact overall survival 

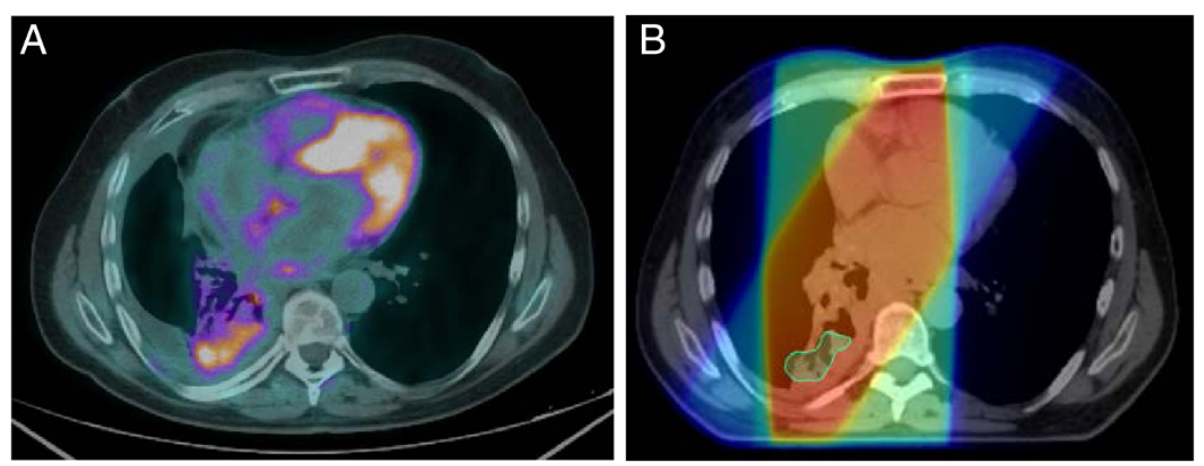

Figure 1 In-field recurrence. A.) PET/CT 1 year post-treatment demonstrating recurrence in the posterior right lung. B.) Region of PET recurrence contoured and fused to original CT simulation scan. Dose wash on CT simulation reveals the recurrence to be an in-field recurrence with a D95\% to the volume of recurrence of $62 \mathrm{~Gy}$.

as demonstrated by a recent meta-analysis comparing concurrent chemoradiation versus sequential chemotherapy and radiation [5]. Phase I and II studies evaluating radiation dose escalation to 74 Gy revealed promising results with significant improvements in progression free survival and overall survival [15-18]. Because of these early promising results, RTOG 06-17 studied the question of dose escalation in a phase III setting. The final results regarding the question of dose were recently presented in abstract form, which revealed a detriment to dose escalation in terms of locoregional failure and overall survival [19]. The LRF rate at 18 months was $35.3 \%$ for the 60 Gy arm and $44 \%$ for the 74 Gy arm. Based on the results of RTOG 06-17, radiation dose escalation at 2 Gy per fraction does not appear to be the answer to improving local control for patients with Stage III NSCLC, and other strategies are needed to optimize locoregional control.

In this study, 55\% of in-field LRFs occurred within the involved mediastinum. Recently, investigators at University of Kentucky reported outcomes of a pilot study evaluating a stereotactic body radiation therapy (SBRT) boost to residual primary lung disease for stage IIA-IIIB NSCLC after 60 Gy of concurrent chemoradiation. Though median follow-up was quite short at 13 months, actuarial local control was $83 \%$ [20]. While this approach may be promising, it does not address the mediastinum, which is the more common site of in-field recurrence. At Emory, a phase I dose escalation study to determine the maximum tolerated dose of a SBRT boost to residual primary lung and mediastinal disease after concurrent chemoradiation to a dose of 44 Gy with standard fractionation is currently ongoing.

The use of FDG PET/CT for patients with NSCLC has dramatically increased over the past two decades. Increasing SUVmax of the primary tumor has been associated with a reduction in disease-free survival and overall survival in patients with early stage NSCLC treated with SBRT [21]. FDG PET will likely yield to the development of imaging biomarkers that can aid in predicting LRF in the locally advanced NSCLC population. Determining if pre-treatment SUVmax or other pre-treatment FDG PET parameters are associated with LRF after definitive chemoradiation for Stage III NSCLC is intriguing as this could lead to tailoring of radiation dose escalation to areas thought to be at higher risk for failure. In this study, the median pre-treatment SUVmax for anatomic
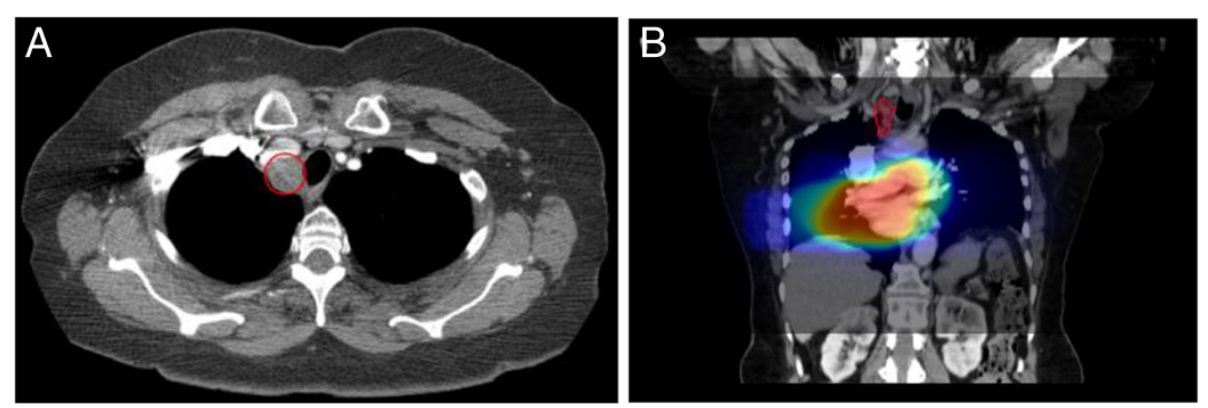

Figure 2 Out-of-field recurrence. A.) CT scan 1 year post-treatment demonstrating a recurrence involving a right paratracheal lymph node. B.) Region of recurrence contoured and fused to original CT simulation scan. Dose wash on CT simulation scan demonstrates an out-of-field recurrence with a D95\% of 1 Gy. 

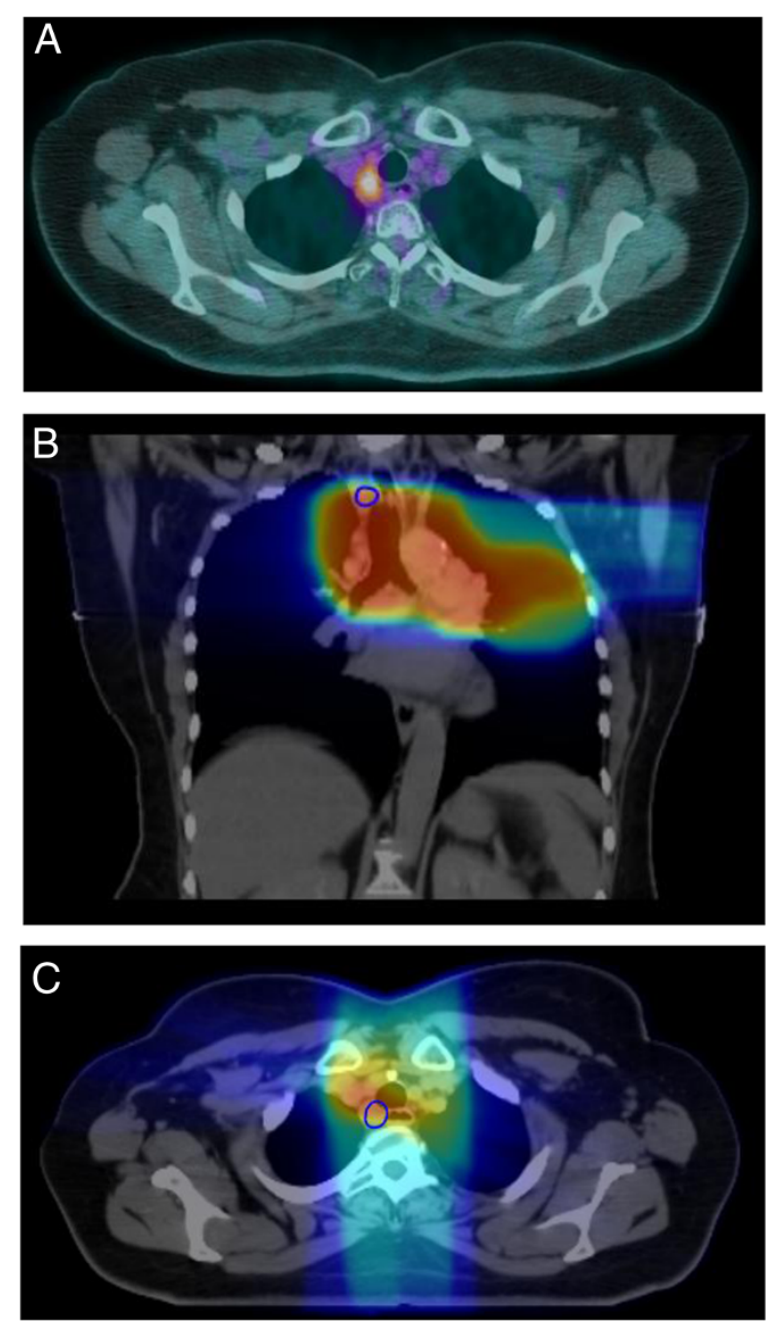

Figure 3 Marginal recurrence. A.) PET/CT 4 months post-treatment demonstrating SUV uptake in a right paratracheal lymph node. B.) and C.) Region of recurrence contoured and fused to original $C T$ simulation scan. Dose wash on CT simulation scan demonstrates a marginal recurrence with a D95\% of $50 \mathrm{~Gy}$ in a coronal view and axial view, respectively.

regions of LRF was 13.4. This SUVmax was not statistically different from the SUVmax of patients who did not develop a LRF. While pre-treatment SUVmax as a potential marker for regions at risk of LRF may not be useful, other PET parameters are currently being explored, including in-treatment FDG/PET CT in the ongoing RTOG 11-06 protocol.

Table 3 All cumulative sites of local regional failure

\begin{tabular}{ll}
\hline Location of local regional failure & $\mathbf{n = 3 9}$ \\
\hline In-Field & $22(56 \%)$ \\
Out-of-field & $13(33 \%)$ \\
Marginal & $4(10 \%)$ \\
\hline
\end{tabular}

\section{Conclusions}

This study demonstrates that in-field recurrence, particularly nodal recurrence, is the primary mode of LRF after definitive radiotherapy for locally advanced NSCLC. Marginal failures are rare and in-field recurrences are receiving the intended radiation dose. Intrinsic radioresistance is likely contributing to persistent LRF despite improvements in radiation techniques. Pretreatment SUVmax was not associated with LRF in this study, but the use of other PET parameters to determine high-risk regions for LRF remains worthy of further investigation.

\section{Abbreviations}

LRF: Locoregional failure; NSCLC: Non-small cell lung cancer; CT: Computed tomography; PET: Positron emission Tomography; SUV: Standardized uptake value; D95: Dose to 95\% of the volume; 3DCRT: 3-dimensional conformal radiation therapy; IMRT: Intensity modulated radiation therapy; FDG: Fluorodeoxyglucose; AP: Anteroposterior; PA: Posteroanterior; GTV: Gross tumor volume; CTV: Clinical target volume; PTV: Planning target volume; KV: Kilovoltage; SBRT: Stereotactic body radiation therapy; RTOG: Radiation Therapy Oncology Group.

\section{Competing interests}

E.S. is entitled to royalties derived from Velocity Medical Solutions' sale of products. The terms of this agreement have been reviewed and approved by Emory University in accordance with its conflict of interest policies. T.F. is entitled to royalties derived from Velocity Medical Solutions' sale of products and serves as a scientific advisor to Velocity Medical Solutions and owns equity in the company. The terms of this agreement have been reviewed and approved by Emory University in accordance with its conflict of interest policies.

There are no other conflicts of interest to report.

\section{Authors' contributions}

RSR carried out the data collection, analysis of results and drafted the manuscript. ES assisted in conception of the study, acquisition of data, and reviewing the manuscript critically. TF assisted in interpretation of data, reviewing the manuscript, and revising the manuscript critically for important intellectual content. LJS and JJB contributed by reviewing the manuscript and revising it critically for important intellectual content. WJC. contributed by analyzing and interpreting the results and reviewing the manuscript critically for important intellectual content. KAH assisted in conception of the project, analysis and interpretation of results, drafting the manuscript, and reviewing it critically for important intellectual content. All authors read and approved the final manuscript.

\section{Authors' information}

R.S.R: Resident Physician, Department of Radiation Oncology, Emory University E.S.: Assistant Professor, Medical Physics, Department of Radiation Oncology, Emory University

T.F.: Director of Medical Physics, Department of Radiation Oncology, Emory University L.J.S.: Assistant Professor, Department of Radiation Oncology, Emory University J.J.B.: Professor, Department of Radiation Oncology, Emory University W.J.C.: Professor and Chair, Department of Radiation Oncology, Emory University K.A.H.: Assistant Professor, Department of Radiation Oncology, Emory University

Received: 12 May 2014 Accepted: 16 August 2014 Published: 26 August 2014

\section{References}

1. Siegel $R$, Naishadham D, Jemal A: Cancer statistics, 2013. CA Cancer J Clin 2013, 63:11-30.

2. Furuse K, Fukuoka M, Kawahara M, Nishikawa H, Takada Y, Kudoh S, Katagami N, Ariyoshi Y: Phase III study of concurrent versus sequential thoracic radiotherapy in combination with mitomycin, vindesine, and cisplatin in unresectable stage III non-small-cell lung cancer. J Clin Oncol 1999, 17:2692-2699. 
3. Curran WJ Jr, Paulus R, Langer CJ, Komaki R, Lee JS, Hauser S, Movsas B, Wasserman T, Rosenthal SA, Gore E, Machtay M, Sause W, Cox JD: Sequential vs. concurrent chemoradiation for stage III non-small cell lung cancer: randomized phase III trial RTOG 9410. J Natl Cancer Inst 2011, 103:1452-1460.

4. Fournel $P$, Robinet $G$, Thomas $P$, Souquet $P J$, Lena $H$, Vergnenegre $A$ Delhoume JY, Le Treut J, Silvani JA, Dansin E, Bozonnat MC, Daures JP, Mornex F, Perol M: Randomized phase III trial of sequential chemoradiotherapy compared with concurrent chemoradiotherapy in locally advanced non-small-cell lung cancer: Groupe Lyon-Saint-Etienne d'Oncologie Thoracique-Groupe Francais de Pneumo-Cancerologie NPC 95-01 Study. J Clin Oncol 2005, 23:5910-5917.

5. Auperin A, Le Pechoux C, Rolland E, Curran WJ, Furuse K, Fournel P, Belderbos J, Clamon G, Ulutin HC, Paulus R, Yamanaka T, Bozonnat MC, Uitterhoeve A, Wang X, Stewart L, Arriagada R, Burdett S, Pignon JP: Meta-analysis of concomitant versus sequential radiochemotherapy in locally advanced non-small-cell lung cancer. J Clin Oncol 2010, 28:2181-2190.

6. Le Chevalier T, Arriagada R, Quoix E, Ruffie P, Martin M, Tarayre M, Lacombe-Terrier MJ, Douillard JY, Laplanche A: Radiotherapy alone versus combined chemotherapy and radiotherapy in nonresectable non-small-cell lung cancer: first analysis of a randomized trial in 353 patients.

J Natl Cancer Inst 1991, 83:417-423.

7. Dwamena BA, Sonnad SS, Angobaldo JO, Wahl RL: Metastases from non-small cell lung cancer: mediastinal staging in the 1990s-meta-analytic comparison of PET and CT. Radiology 1999, 213:530-536.

8. Marom EM, McAdams HP, Erasmus JJ, Goodman PC, Culhane DK, Coleman RE, Herndon JE, Patz EF Jr: Staging non-small cell lung cancer with whole-body PET. Radiology 1999, 212:803-809.

9. Bradley J, Bae K, Choi N, Forster K, Siegel BA, Brunetti J, Purdy J, Faria S, Vu T, Thorstad W, Choy H: A phase II comparative study of gross tumor volume definition with or without PET/CT fusion in dosimetric planning for non-small-cell lung cancer (NSCLC): primary analysis of Radiation Therapy Oncology Group (RTOG) 0515. Int J Radiat Oncol Biol Phys 2012, 82:441.e431.

10. Vansteenkiste JF, Stroobants SG, Dupont PJ, De Leyn PR, Verbeken EK, Deneffe GJ, Mortelmans LA, Demedts MG: Prognostic importance of the standardized uptake value on (18)F-fluoro-2-deoxy-glucose-positron emission tomography scan in non-small-cell lung cancer: An analysis of 125 cases. Leuven Lung Cancer Group. J Clin Oncol 1999, 17:3201-3206.

11. Berghmans T, Dusart M, Paesmans M, Hossein-Foucher C, Buvat I, Castaigne C, Scherpereel A, Mascaux C, Moreau M, Roelandts M, Alard S, Meert AP, Patz EF Jr, Lafitte JJ, Sculier JP: Primary tumor standardized uptake value (SUVmax) measured on fluorodeoxyglucose positron emission tomography (FDG-PET) is of prognostic value for survival in non-small cell lung cancer (NSCLC): a systematic review and meta-analysis (MA) by the European Lung Cancer Working Party for the IASLC Lung Cancer Staging Project. J Thorac Oncol 2008, 3:6-12.

12. Badea $C T$, Schreibmann $E$, Fox $T$ : $A$ registration based approach for $4 D$ cardiac micro-CT using combined prospective and retrospective gating. Med Phys 2008, 35:1170-1179.

13. Kirby N, Chuang C, Ueda U, Pouliot J: The need for application-based adaptation of deformable image registration. Med Phys 2013, 40:011702.

14. Li T, Thorndyke B, Schreibmann E, Yang Y, Xing L: Model-based image reconstruction for four-dimensional PET. Med Phys 2006, 33:1288-1298.

15. Bradley JD, Bae K, Graham MV, Byhardt R, Govindan R, Fowler J, Purdy JA, Michalski JM, Gore E, Choy H: Primary analysis of the phase II component of a phase I/II dose intensification study using three-dimensional conformal radiation therapy and concurrent chemotherapy for patients with inoperable non-small-cell lung cancer: RTOG 0117. J Clin Oncol 2010, 28:2475-2480.

16. Schild SE, McGinnis WL, Graham D, Hillman S, Fitch TR, Northfelt D, Garces YI, Shahidi H, Tschetter LK, Schaefer PL, Adjei A, Jett J: Results of a Phase I trial of concurrent chemotherapy and escalating doses of radiation for unresectable non-small-cell lung cancer. Int J Radiat Oncol Biol Phys 2006, 65:1106-1111

17. Socinski MA, Blackstock AW, Bogart JA, Wang X, Munley M, Rosenman J, Gu L, Masters GA, Ungaro P, Sleeper A, Green M, Miller AA, Vokes EE: Randomized phase II trial of induction chemotherapy followed by concurrent chemotherapy and dose-escalated thoracic conformal radiotherapy (74 Gy) in stage III non-small-cell lung cancer: CALGB 30105. J Clin Oncol 2008, 26:2457-2463.
18. Stinchcombe TE, Lee CB, Moore DT, Rivera MP, Halle J, Limentani S, Rosenman JG, Socinski MA: Long-term follow-up of a phase $1 /$ II trial of dose escalating three-dimensional conformal thoracic radiation therapy with induction and concurrent carboplatin and paclitaxel in unresectable stage IIIA/B non-small cell lung cancer. J Thorac Oncol 2008, 3:1279-1285.

19. Bradley JD, Paulus R, Komaki R, Masters GA, Forster K, Schild SE, Bogart J, Garces YI, Narayan S, Kavadi V, Nedzi LA, Michalski JM, Johnson D, MacRae RM, Curran WJ, Choy H: A Randomized Phase III Comparison of Standard-Dose (60 Gy) Versus High-Dose (74 Gy) Conformal Chemoradiotherapy With or Without Cetuximab for Stage III non-Small Cell Lung Cancer: Results on Radiation Dose in RTOG 0617. J Clin Oncol 2013, 31. (suppl; abstr 7501) Abstract Presentation. ASCO 2013.

20. Feddock J, Arnold SM, Shelton BJ, Sinha P, Conrad G, Chen L, Rinehart J, McGarry RC: Stereotactic body radiation therapy can be used safely to boost residual disease in locally advanced non-small cell lung cancer: a prospective study. Int J Radiat Oncol Biol Phys 2013, 85:1325-1331.

21. Takeda A, Sanuki N, Fujii H, Yokosuka N, Nishimura S, Aoki Y, Oku Y, Ozawa Y, Kunieda E: Maximum standardized uptake value on FDG-PET is a strong predictor of overall and disease-free survival for non-small-cell lung cancer patients after stereotactic body radiotherapy. J Thorac Oncol 2014, 9:65-73.

\section{doi:10.1186/1748-717X-9-187}

Cite this article as: Rajpara et al:: Locoregional tumor failure after definitive radiation for patients with stage III non-small cell lung cancer. Radiation Oncology 2014 9:187.

\section{Submit your next manuscript to BioMed Central and take full advantage of:}

- Convenient online submission

- Thorough peer review

- No space constraints or color figure charges

- Immediate publication on acceptance

- Inclusion in PubMed, CAS, Scopus and Google Scholar

- Research which is freely available for redistribution
C) Biomed Central 